\title{
On the Performance of ISFET-Based Device for Water Quality Monitoring
}

\author{
Pawan Whig, Syed Naseem Ahmad \\ Research Scholar, Department of Electronics and Communication Engineering, \\ Jamia Millia Islamia, New Delhi, India \\ E-mail: pawanwhig@gmail.com, snahmad@jmi.ac.in \\ Received September 7, 2011; revised October 4, 2011; accepted October 20, 2011
}

\begin{abstract}
A new configuration realizing water quality monitoring device using ISFET involving low power CMOS Integrated "Ion Sensitive Field Effect transistor (ISFET) - Operational Amplifier is presented. The study's main focus is on simulation of power and performance analysis of ISFET device, which is used for water quality monitoring. This approach can improve calibration of device to a fairly wide range without the use of a high speed digital processor. The conventional device has a drawback of slow slew rate but in this novel design, the device has a better slew rate. A new slew rate enhancement (SRE) incorporated into a ISFET, which does not affect the small signal frequency response. The functionality of the circuit is tested using Tanner simulator version 15 for a $70 \mathrm{~nm}$ CMOS process model also the transfer function realization is done on MATLAB R2011a version, the Very high speed integrated circuit Hardware description language (VHDL) code for the same scheme is simulated on Xilinx ISE 10.1 and various simulation results are obtained. Simulation results are included to demonstrate the results.
\end{abstract}

Keywords: ISFET, Slew Rate, Calibration, Simulation, Monitoring Applications, pH Value, Alkalinity

\section{Introduction}

Monitoring the $\mathrm{pH}$ of water resources and sewage discharge for water pollution is typical and necessary task in today's overdeveloped scenario. The normal $\mathrm{pH}$ for surface water systems is $6.5-8.5$ and for ground water system $6-8$. Water with low $\mathrm{pH}$ is acidic, corrosive and contains several toxic materials which are very dangerous for health, but the water having $\mathrm{pH}$ more than 8.5 is called hard water which does not contain harmful materials but the long use of such kind of water can cause aesthetic problems [1-3]. With the invention of ISFET [4] there has been a rapid development of $\mathrm{pH}$ Measurement Instruments [5]. With the further advancement of semiconductor technology ISFET emerged as a standard device. In spite of the fact that ISFET Sensor has been developed 30 years ago [6], several drawback of ISFET sensor remained unsolved, such as phenomena of fluctuation with time and temperature variations. This cause in drift in the $\mathrm{pH}$ values [7], and result in poor and slow response [8] of the device. The second phenomenon is $\mathrm{pH}$ dependent Temperature Coefficient [9] and non linear temperature dependent mobility in MOSFETS of
ISFET device [10]. Also it was observed that in ISFET drift rate has an exponential incremental tendency with $\mathrm{pH}$ values as well as Temperature.

In Urban water supply system, the water quality determining indices such as $\mathrm{pH}$ value and turbidity are monitored continuously. When the indices exceed the limiting value, the system will effectively handle the treatment against deterioration ensuring the safety of water. Water is vital for all known forms of life. Many research works have contributed to design water quality measureing devices. But it has always been a challenge to find a precise and accurate device for monitoring the quality of water.

The concept of $\mathrm{pH}$ was first introduced by Danish chemist Soren Peder Lauritz Sorensen at the Carlsberg Laboratory in 1909 and revised to the modern $\mathrm{pH}$ in 1924 after it became apparent that electromotive force in cells depended on activity rather than concentration of hydrogen ions. The $\mathrm{pH}$ is a measure of the acidity or basicity of an aqueous solution.

The use of micro sensors for infield monitoring of environmental parameters is gaining interest due to their advantages over conventional sensors. In the field of 
micro sensors for environmental applications, Ion Selective Field Effect Transistors (ISFETs) has proved to be of special application. They are particularly helpful for measuring $\mathrm{pH}$ and other ions in small volumes and they can be integrated in compact flow cells for continuous measurements and monitoring [11-16].

Pure water is said to be neutral, with a $\mathrm{pH}$ close to 7.0 at $25^{\circ} \mathrm{C}(77 \mathrm{~F})$. Solutions with a $\mathrm{pH}$ less than seven (7) are said to be acidic and solutions with a $\mathrm{pH}$ greater than seven (7) are basic or alkaline.

This study highlights a performance analysis of low power CMOS Integrated "Ion Sensitive Field Effect transistor (ISFET) - Operational Amplifier. The studies mainly focus on the simulation of power and performance analysis of ISFET device, which is used for water quality monitoring applications [17-23]. This approach can improve calibration of device to a fairly wide range without the use of a high speed digital processor. The conventional device generally used, consumes high power and is not stable with temperature and frequency variations for long term monitoring. The conventional device has a drawback of slow slew rate but in this novel design the device has a fairly good slew rate this device has a simple architecture, and hence is very suitable for the water quality monitoring application. In this novel design, the device is free from noise and other effect and is seen consuming low power of the order of $13 \mu \mathrm{W}$.

The paper is organized as follows: Section 2 describes the ISFET, Section 3 explains the device description and mathematical modeling and, Section 4 Simulation and result analysis, Section 5 gives the results and conclusions and Section 6 present the future works to be done.

\section{ISFET}

An ISFET is an ion-sensitive field-effect transistor which has a property of measuring ion concentrations in solution; when the ion concentration (such as $\mathrm{H}^{+}$) changes, the current through the transistor will change accordingly [5]. Here, the solution is used as the gate electrode. A voltage between substrate and oxide surfaces arises due to an ions' sheath.

The ISFET has the similar structure as that of the MOSFET except that the poly gate of MOSFET is removed from the silicon surface and is replaced with a reference electrode inserted inside the solution, which is directly in contact with the hydrogen ion $\left(\mathrm{H}^{+}\right)$sensitive gate electrodeas shown in Figure 1 [6].

At the interface between gate insulator and the solution, there is an electric potential difference that depends on the concentration of $\mathrm{H}^{+}$of the solution, or so called, $\mathrm{pH}$ value. The variation of this potential caused by the
$\mathrm{pH}$ variation will lead to modulation of the drain current [7]. As a result, the Id-Vgs transfer characteristic of the ISFET, working in triode region, can be observed similar with that of MOSFET:

$$
I_{d s}=\frac{\mu C_{o x} W}{L}\left[\left(V_{g s}-V_{t h \_i s f e t}\right) V_{D S}-\frac{1}{2}\left(V_{d s}\right)^{2}\right]
$$

The threshold voltage is only different in case of MOSFET. In ISFET, defining the metal connection of the reference electrode as a remote gate, the threshold voltage is given by:

$$
\begin{aligned}
V_{t h(\mathrm{ISFET})}= & E_{R e f}+\Delta \Phi^{1 j}-\Psi_{e o l}+\chi^{s o l}+\frac{-\Phi s}{q} \\
& -\frac{Q o x+Q S S}{C_{o x}}+\gamma\{2 \varphi £\}^{1 / 2}+2 \Phi_{£}
\end{aligned}
$$

where $E_{R e f}$ is Potential of reference electrode, $\Delta \Phi^{1 j}$ is the potential drop between the reference electrode and the solution, which typically has a value of $3 \mathrm{mV}$ [8]. $\Psi_{e o l}$ is the potential which is $\mathrm{pH}$-independent; it can be viewed as a common-mode input signal for an ISFET interface circuit in any $\mathrm{pH}$ buffer solution and can be nullified during system calibration and measurement procedures with a typical value of $50 \mathrm{mV}$ [9]. $\chi^{\text {sol }}$ is the surface dipole potential of the solvent being independent of $\mathrm{pH}$.

The terms in the parentheses are almost the same as that of the MOSFET threshold voltage except that of absence of the gate metal function. The other terms in above equation are a group of chemical potential, among which the only chemical input parameter shown has to be a function of solution $\mathrm{pH}$ value. This chemical dependent

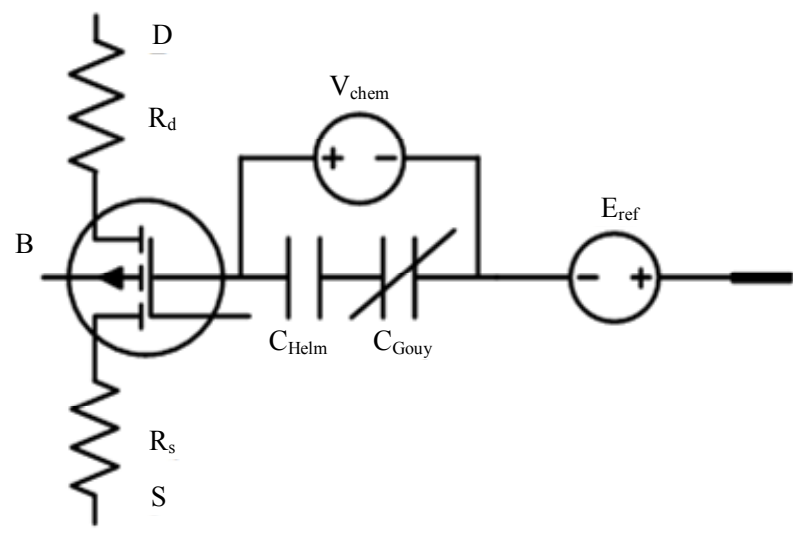

Figure 1. Sub circuit block of ISFET macro model.

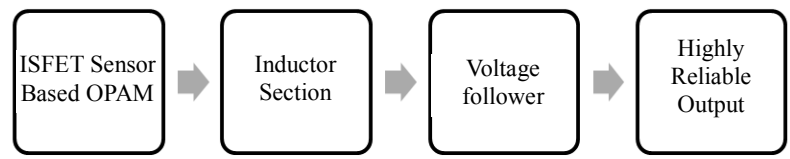

Figure 2. Block diagram of monitoring device. 
characteristic has already been explained by the Hal and Eijkel's theory [15] which is elaborated using the general accepted site-binding model and the Gouy-ChapmanStern model.

\section{Device Description and Mathematical Modeling}

The basic structure of the device consists of three major parts, (I) ISFET (II) Inductor section, and (III) voltage follower, as shown in Figure 2. The proposed circuit of device is shown above, in which the output of the ISFET sensor is fed into one of the terminal of the voltage follower, which helps from the loading effect and keeping the voltage level constant irrespective of the change in the current value. This practise increases the sensitivity of the sensor, and even a very small value can be observed at the output.

As

$$
V_{o}=V_{i n}+\frac{R_{f}}{R_{i}}
$$

Under ideal condition the Op-Amp $R_{i}=\infty$ and thus

$$
V_{o}=V_{\text {in }}
$$

\section{ISFET Sensor Based Op-Amp}

The threshold voltage of a dual dielectric gate ISFET is given by

$$
\begin{aligned}
V_{T H} & =\phi_{E S}-\frac{Q_{S S}}{C_{i n}}-\frac{Q_{l}}{C_{l}}+2 \phi_{F}-\frac{\sqrt{2 K_{s} \in_{0} q N_{B}\left|2 \phi_{F}+V_{S B}\right|}}{C_{i n}} \\
& +2.303 \frac{k T}{q} \cdot S \cdot\left(\mathrm{pH}-\mathrm{pH}_{\mathrm{pzc}}\right)
\end{aligned}
$$

where $\phi_{E S}$ is the "work function" difference' between the electrode in contact with the electrolyte and the semiconductor $=\phi_{i}$, is the interface charge sandwiched between the dual dielectric of the ISFET gate $Q_{S S}$, is a lumped interface and fixed charge referred to the oxide/silicon interface $C_{l}$ is the top gate dielectric capacitance per unit area, $C_{i n}$ is the total gate capacitance per unit area, $N_{B}$ is the bulk doping, $V_{S B}$ is the source-to-bulk reverse bias, $\phi_{F}$ is the Fermi-level of the silicon bulk, $S$ is the sensitivity factor of the top $\mathrm{pH}$ sensing insulator and $\mathrm{pH}_{\mathrm{pZc}}$ is the point-of-zero-charge of the sensing insulator of the ISFET.

The circuit functions as follows: when the ISFEToperational amplifier is configured as a voltage follower as shown in Figure 3 the output voltage $\left(V_{o}\right)$ is equal to the input voltage $\left(V_{i n}\right)$; any difference in threshold voltages and bias currents between the two input transistors at the differential input stage will also appear at the output. The output voltage of the device is found to be

$$
V_{o}=V_{i n}+\phi_{m s}-\phi_{E S}-2.303 \frac{k T}{q} \cdot S \cdot\left(p H-p H_{p z c}\right)+V_{o s}
$$

where $V_{\text {in }}$ is the offset voltage which is temperature and light sensitive but chemically insensitive. The offset: voltage includes terms arising from the mismatch of the total gate capacitances $\left(\mathrm{C}_{\text {in }}\right)$, semiconductor bulk charges $\sqrt{2 K_{s} \in_{0} q N_{B}\left|2 \phi_{F}+V_{S B}\right|}$, insulator interface charges $\left(Q_{s s}, Q_{l}\right)$, and transistor gain $(\beta)$ between the MOSFET and the ISFET.

The ideal reference electrode commonly employed in combination with the ISFET sensor as shown in Figure 4 serves two functions:

1) to provide an electrical contact to the test electrolyte and thus define the electrical potential of the electrolyte;

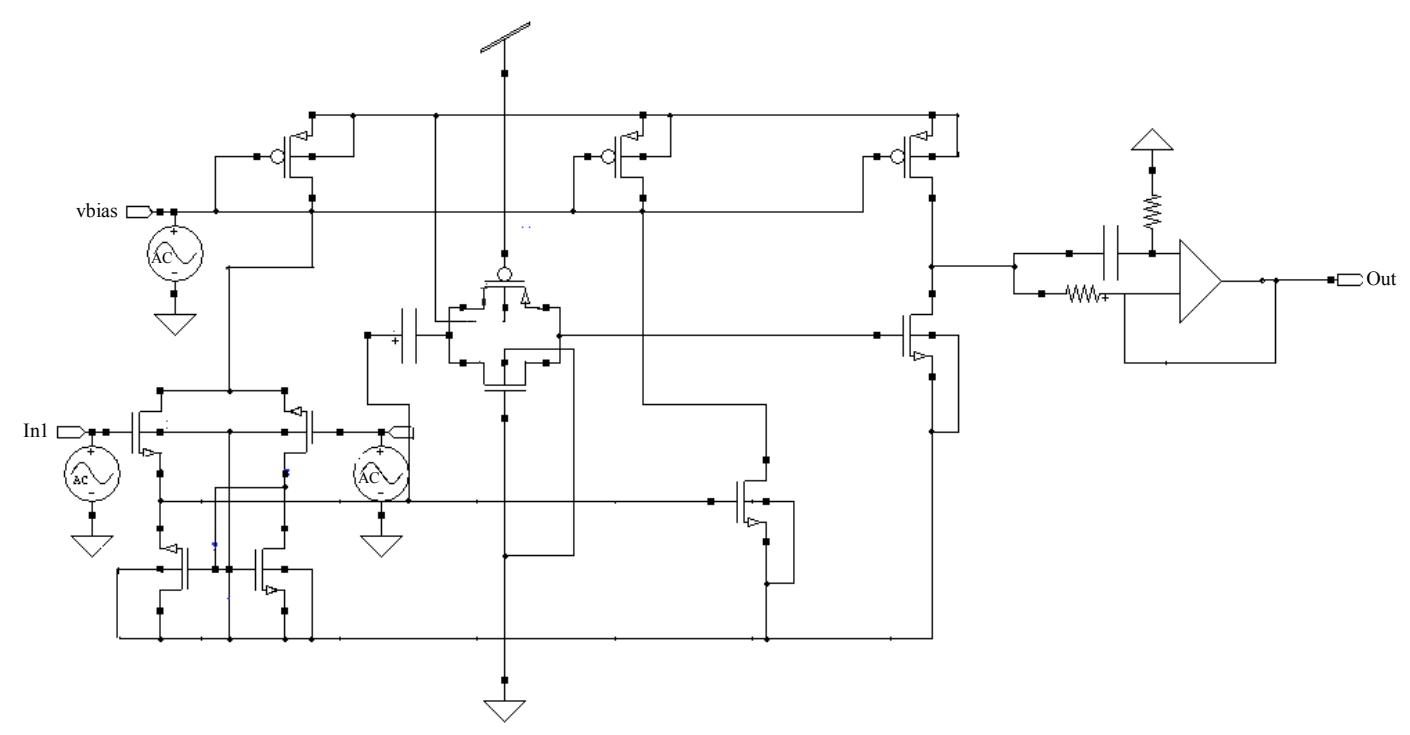

Figure 3. Equivalent circuit of device. 


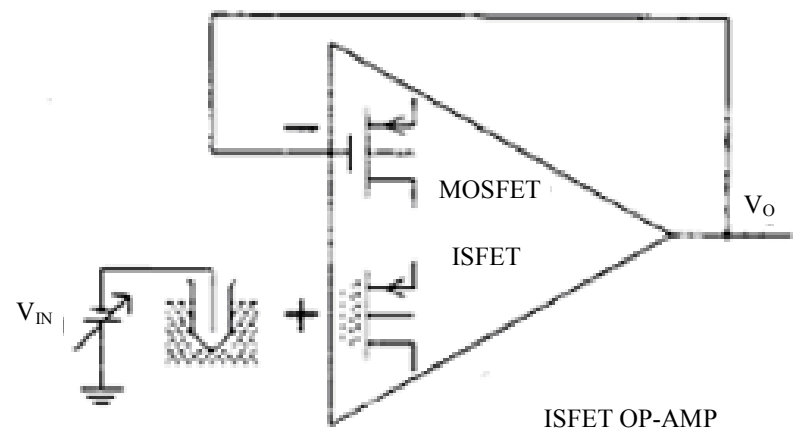

Figure 4. Block diagram Representation of ISFET based OP-amp.

and 2) to provide an electrode-electrolyte interface potential invariant with the electrolyte composition such that the dependence of the threshold voltage of the ISFET on the electrolyte composition arises only from the electrolyte-insulator interface of the ISFET.

\section{Simulation and Result Analysis}

The circuit of the Op-amp based Ion Sensitive Field Effect Transistor (ISFET) is implemented on Tanner tool version-15. The device is modeled on $250 \mathrm{~nm}$ technology as shown in Figure 5 and the output results of T-spice command file and output waveform of the transient analysis are found and shown in Figures 6 and 7. From the transient analysis it is observed that the output waveform is not linear. This shows that the slew rate of the device is poor. To improve the slew rate, a simulated inductor is placed at the output and the analysis results as computed are shown in the Figures 8-10. It may be seen that there is a significant improvement in the slew rate when a simulated inductor is placed parallel to the load at the output. Figures 11-13 show the RTL diagram of the device, the components used in device, VHDL instantiation created from source file results obtained when the VHDL code of the device is simulated on Xilinx ISE 10.1. The synthesis file and power result obtained by simulation of the circuit is shown in appendix at the end of the paper. In this proposed design, the device is free from interference effects. It also consumes much low power, in order of $13 \mu \mathrm{W}$. The output observed in Figure 9. is highly linear, indicating that the device is stable for a large dynamic range of input signals.

In the above Table 1 shows the various readings of the device during the transient analysis.

Table 2 Shows the various process parameters used in the proposed scheme and it is observed that the Averge power consumed by the device is $13 \mu \mathrm{W}$ and the max power consumed is $16 \mu \mathrm{W}$ under the current range of 1 $\mu \mathrm{A}-50 \mu \mathrm{A}$.

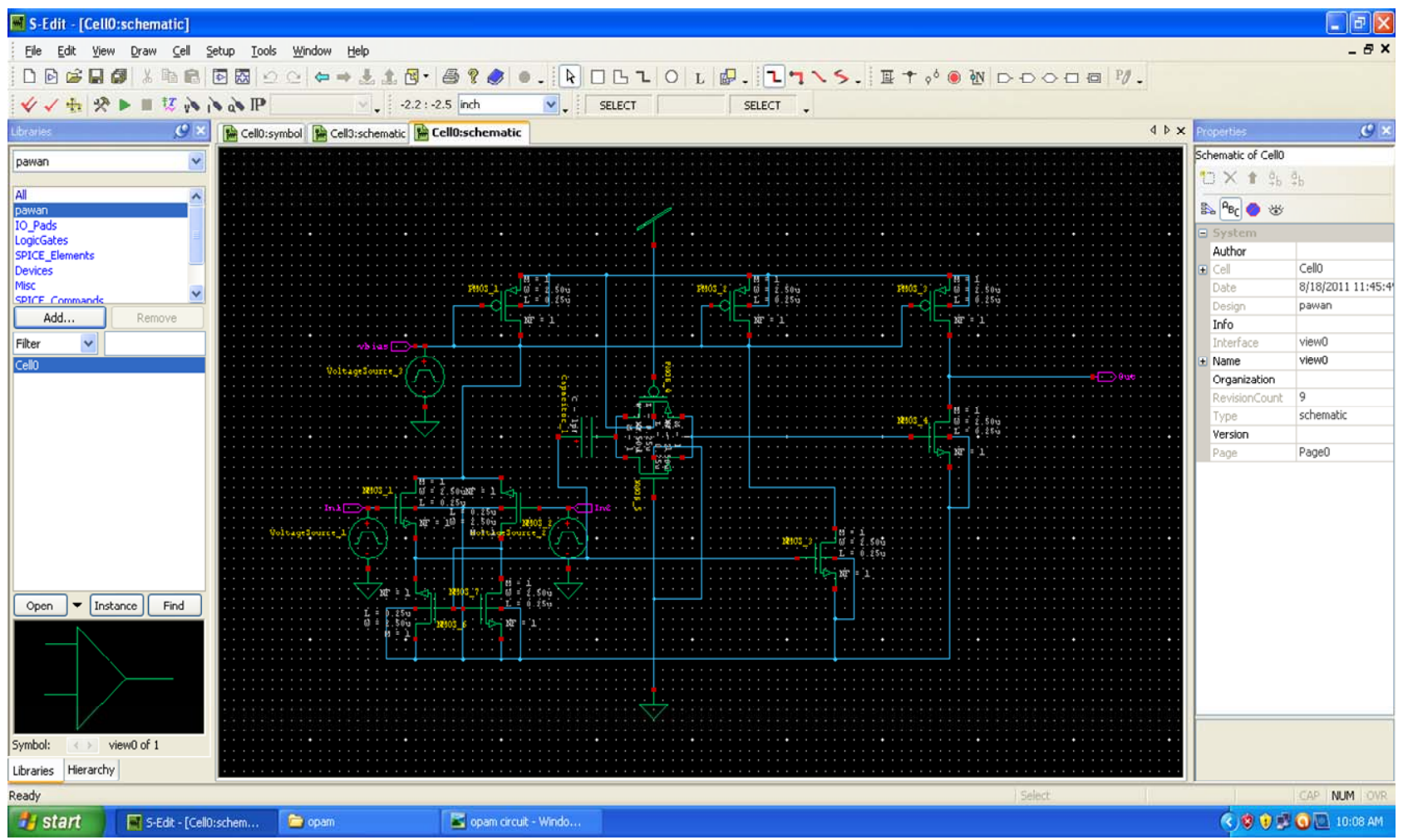

Figure 5. Circuit diagram of device. 


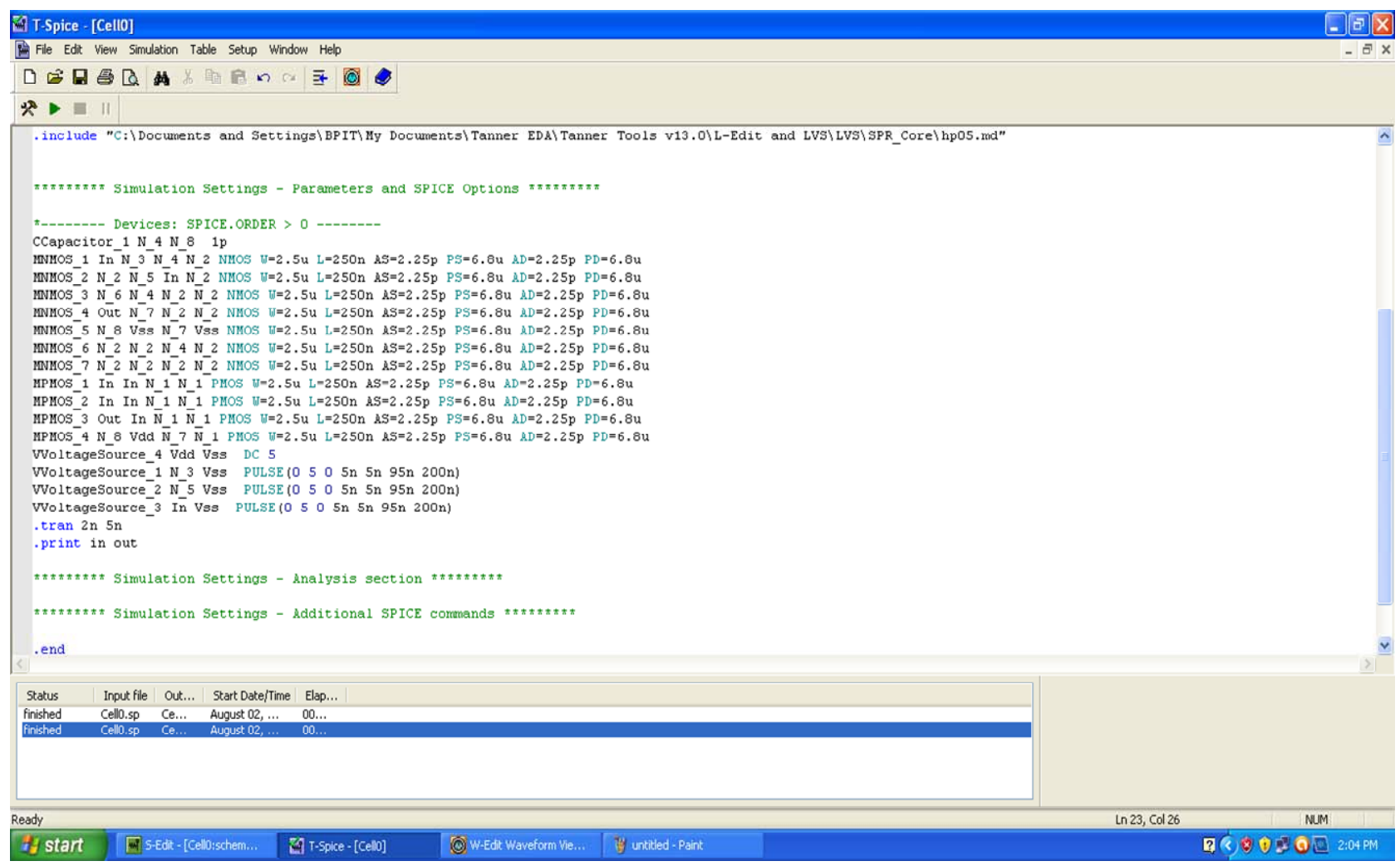

Figure 6. T-Spice file for the above circuit.

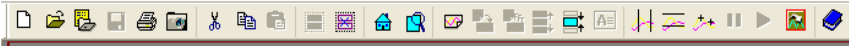

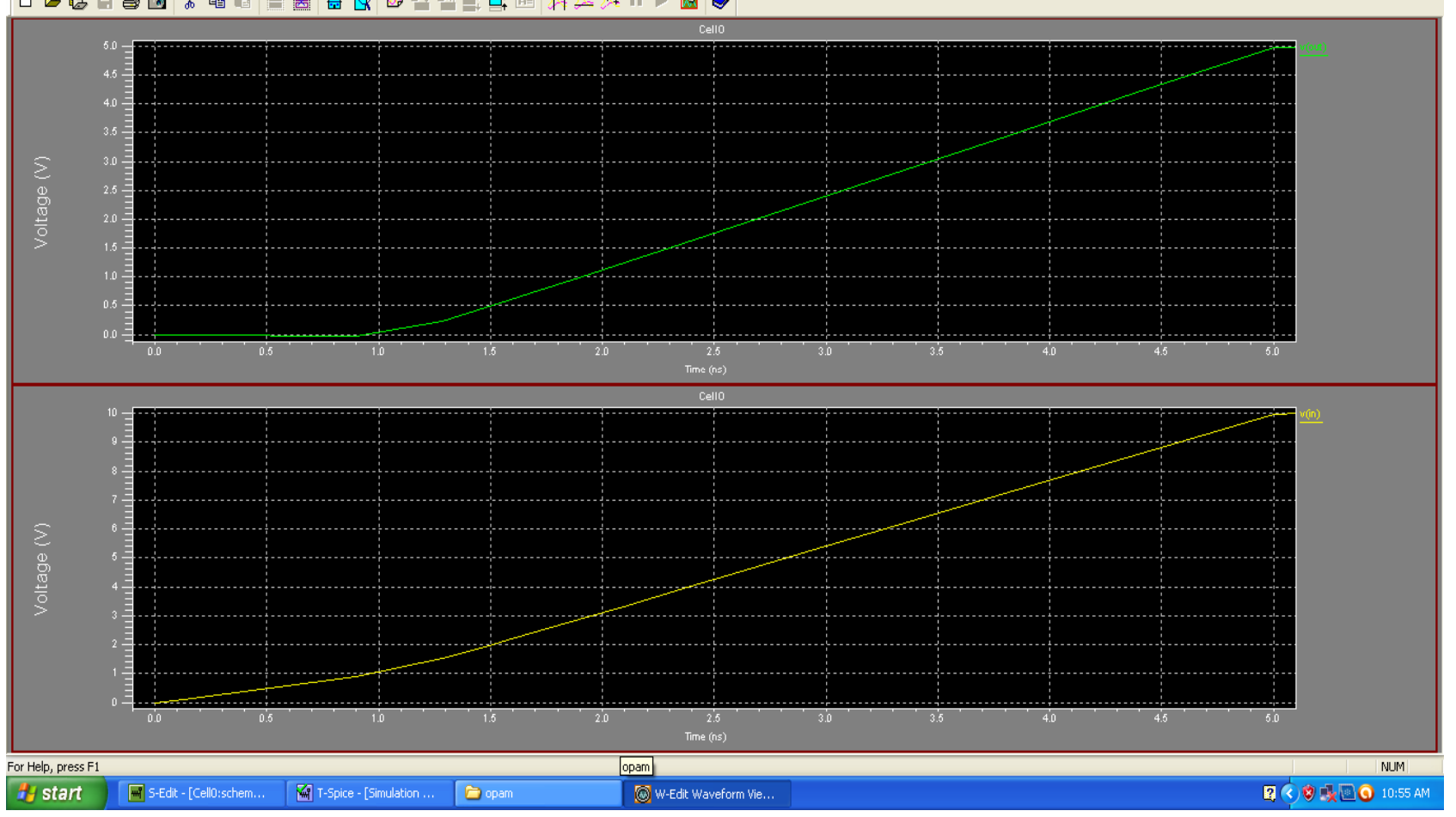

Figure 7. Output waveform of the above circuit. 


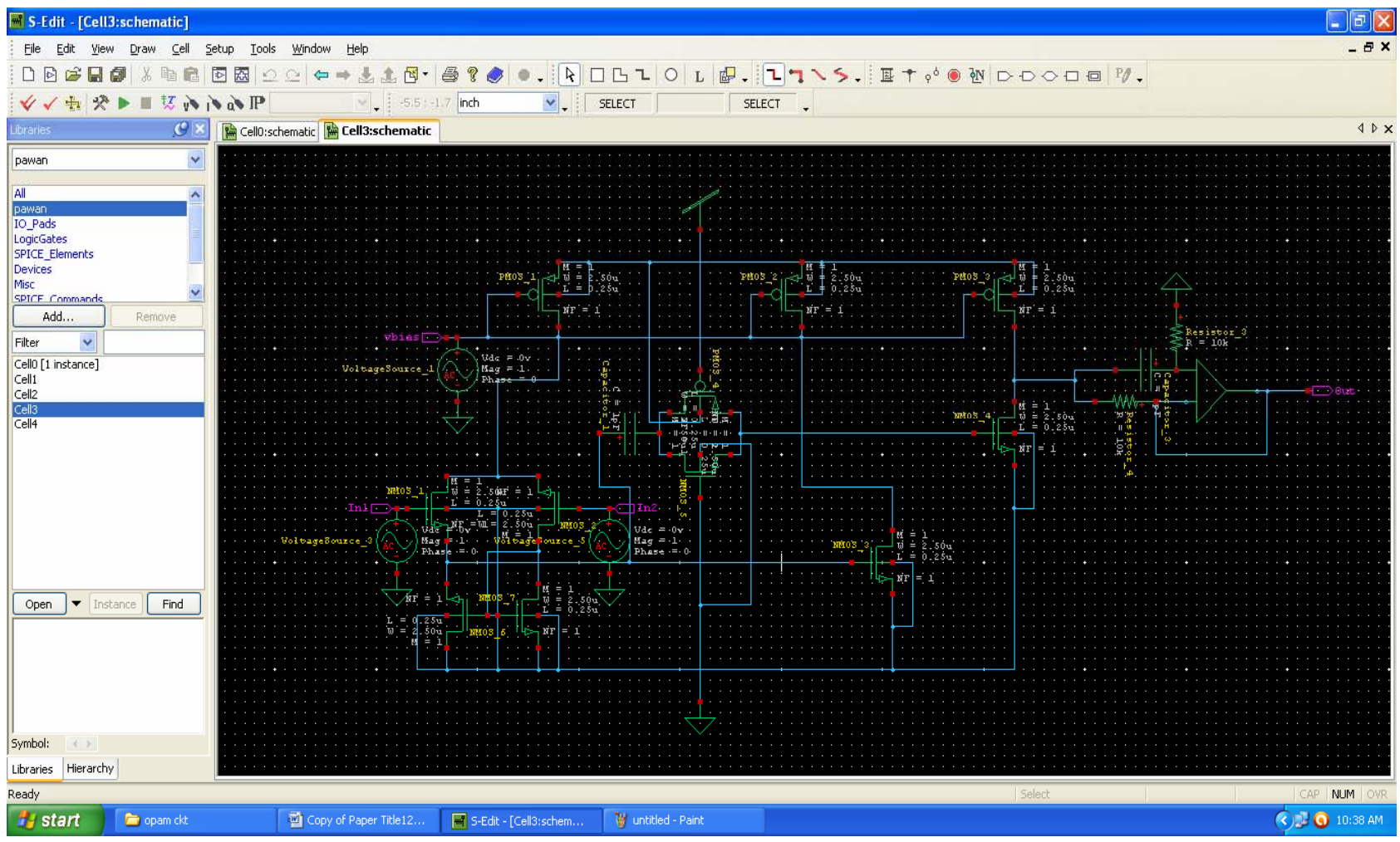

Figure 8. Circuit diagram of device using inductor.

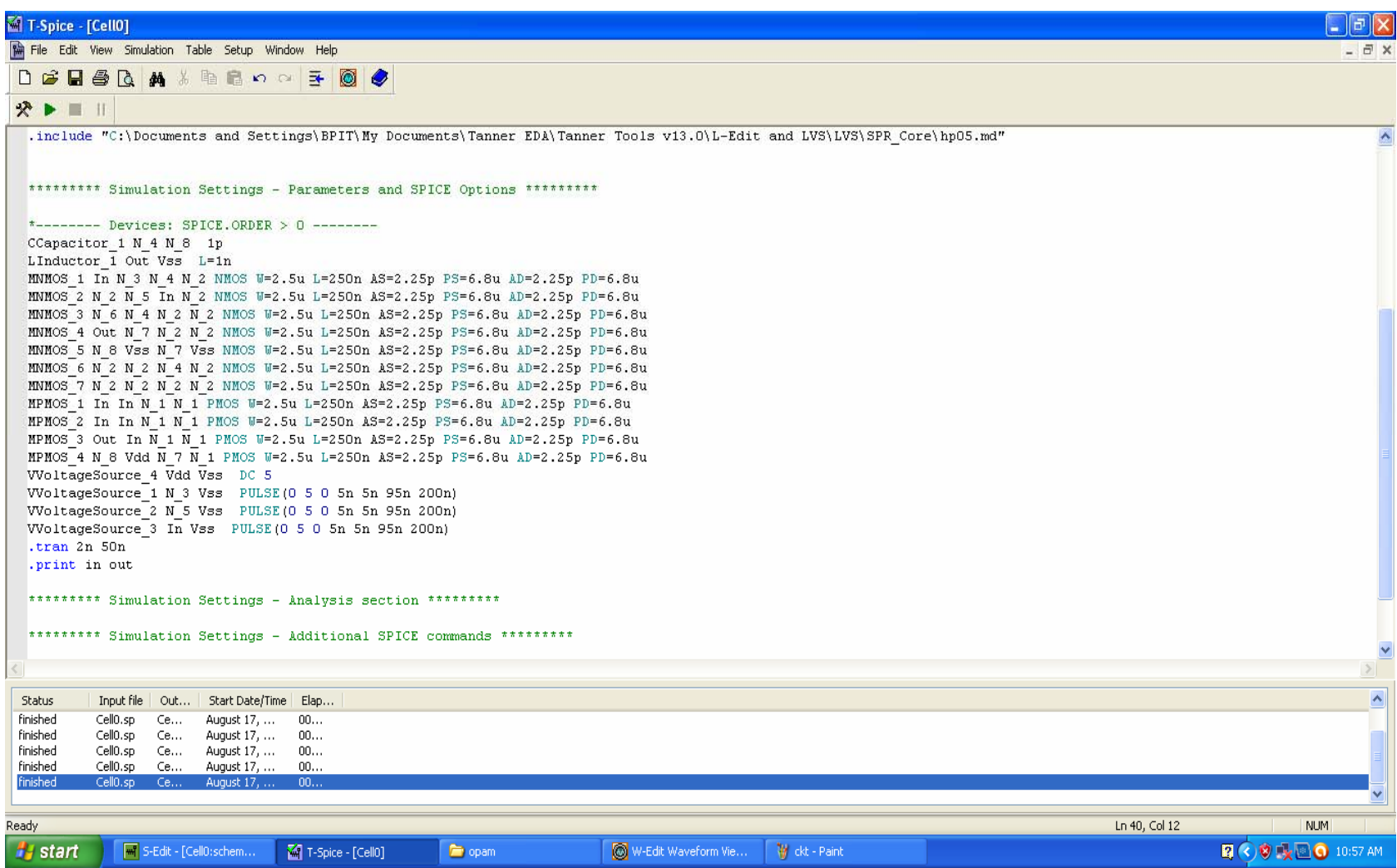

Figure 9. T-Spice file for the above circuit. 
O) W-Edit Waveform Viewer - [C:DOCUME-11BPITLOCALS-11TemplCell4.out]

- File Edit View Chart Options Window Help

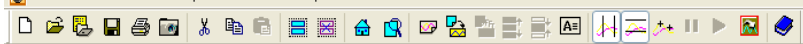

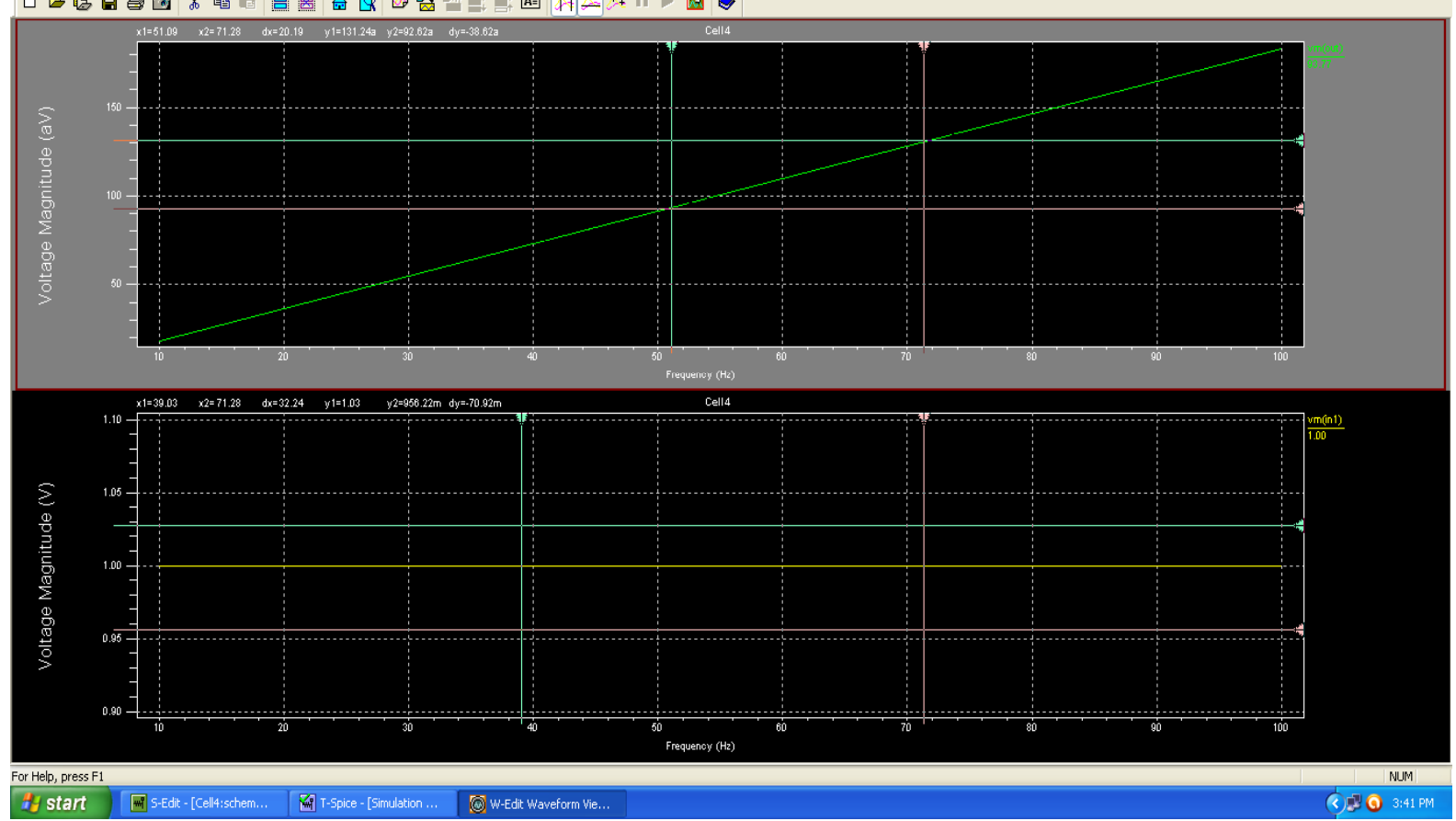

Figure 10. Output waveform of device using simulated inductor.

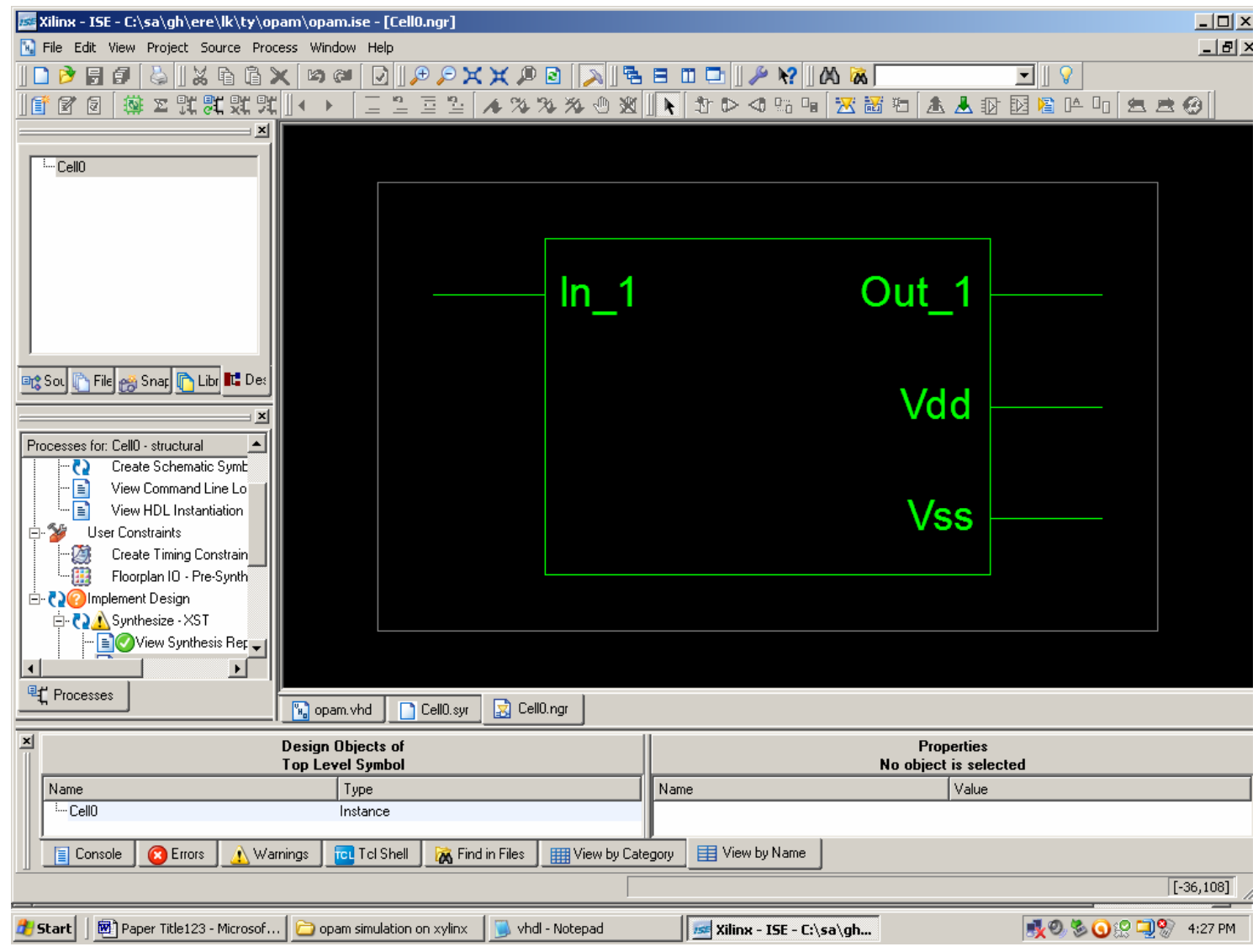

Figure 11. RTL diagram of device. 


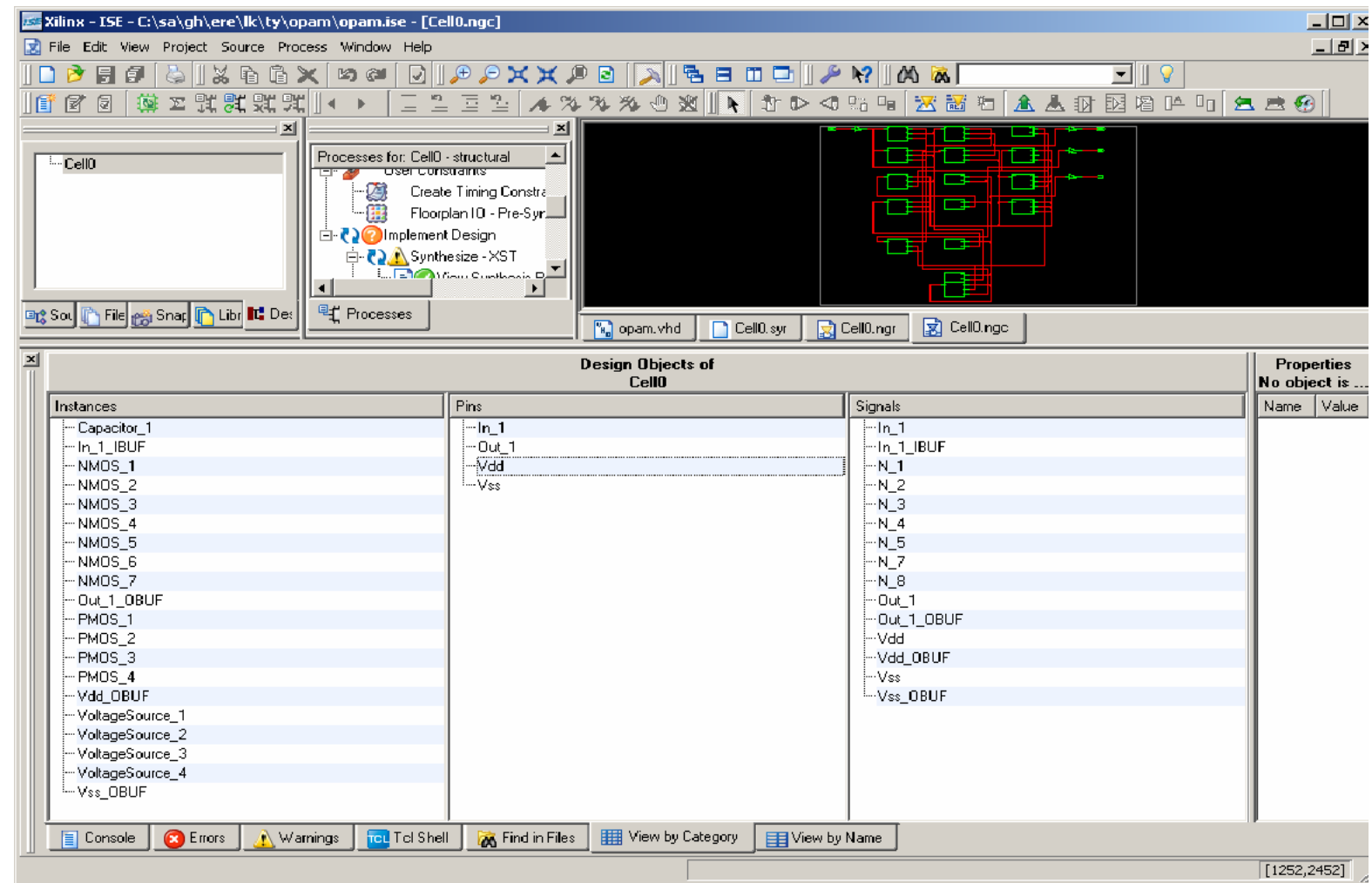

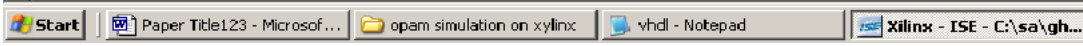

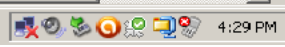

Figure 12. Objects used in the device.

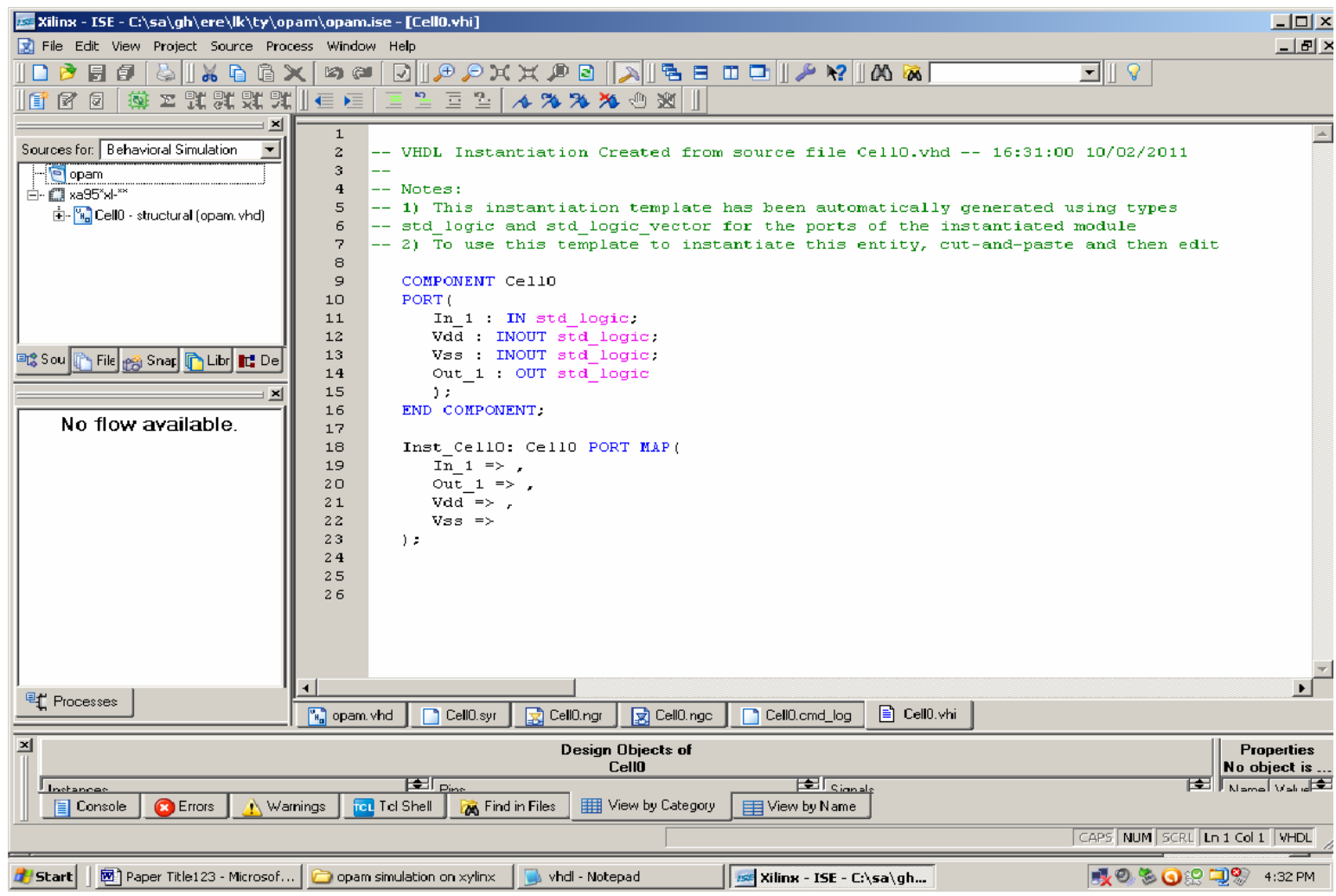

Figure 13. VHDL instantiation created from source file. 
Table 1. Transient analysis.

\begin{tabular}{|c|c|c|}
\hline Time $<\mathrm{s}>$ & $\mathrm{V}($ in 1$)<\mathrm{V}>$ & $\mathrm{V}($ out $)<\mathrm{V}>$ \\
\hline $0.000000 \mathrm{e}+000$ & $0.0000 \mathrm{e}+000$ & $0.0000 \mathrm{e}+000$ \\
\hline $1.250000 \mathrm{e}-010$ & $1.2500 \mathrm{e}-001$ & $9.5606 \mathrm{e}-002$ \\
\hline $6.786460 \mathrm{e}-010$ & $6.7865 \mathrm{e}-001$ & $5.4378 \mathrm{e}-001$ \\
\hline $9.554690 \mathrm{e}-010$ & $9.5547 \mathrm{e}-001$ & $7.8071 \mathrm{e}-001$ \\
\hline $1.093880 \mathrm{e}-009$ & $1.0939 \mathrm{e}+000$ & $8.39976 \mathrm{e}-001$ \\
\hline $1.316775 \mathrm{e}-009$ & $1.3168 \mathrm{e}+000$ & $1.0922 \mathrm{e}+000$ \\
\hline $1.600582 \mathrm{e}-009$ & $1.6006 \mathrm{e}+000$ & $1.3394 \mathrm{e}+000$ \\
\hline $1.922991 \mathrm{e}-009$ & $1.9230 \mathrm{e}-000$ & $1.6229 \mathrm{e}-000$ \\
\hline $2.303210 \mathrm{e}-009$ & $2.3032 \mathrm{e}+000$ & $1.9608 \mathrm{e}+000$ \\
\hline $2.758169 \mathrm{e}-009$ & $2.7582 \mathrm{e}+000$ & $2.3703 e+000$ \\
\hline $3.296876 \mathrm{e}-009$ & $3.2969 \mathrm{e}+000$ & $2.8964 \mathrm{e}+000$ \\
\hline $3.931074 \mathrm{e}-009$ & $3.9311 \mathrm{e}+000$ & $3.4780 \mathrm{e}+000$ \\
\hline $4.678083 e-009$ & $4.6781 \mathrm{e}+000$ & $4.2192 \mathrm{e}+000$ \\
\hline $5.000000 \mathrm{e}-009$ & $5.0000 \mathrm{e}+000$ & $4.5821 \mathrm{e}+000$ \\
\hline
\end{tabular}

Table 2. Device process parameters.

\begin{tabular}{cc}
\hline \multicolumn{2}{c}{ Process Parameters } \\
\hline Power Supply & $5 \mathrm{~V}$ \\
Load Regulation & 3.93 \\
Line Regulation & $0.6 \mathrm{~m}$ \\
Current Range & $1 \mu \mathrm{A}-50 \mu \mathrm{A}$ \\
Average Power Consumed & $13 \mu \mathrm{W}$ \\
Max power & $16 \mu \mathrm{W}$ \\
\hline
\end{tabular}

\section{Conclusions}

A new slew rate enhancement (SRE) circuit which is targeted for ISFET driving with large capacitive load has been presented in this paper. In the proposed design, the device is free from interference effects and seen consuming much low power, in order of $13 \mu \mathrm{W}$. There is the significant improvement in the slew rate when a simulated Inductor is placed parallel to the load at the output. The output. is highly linear, indicating that the device is stable. Both simulation and power results obtained on Xilinx ISE 10.1 and Tanner Tool-15 justify a significant improvement in slew rate and power consumption by using the proposed SRE circuit.

\section{Future work}

This study can be extended and more improvement in terms of power and size can be achieved at layout level and thus more effective results can be obtained.

\section{References}

[1] C. Jimenez-Jorquera, J. Orozoo and A. Baldi, "ISFET Based Microsensors for Environmental Monitoring," Journal of Sensors, Vol. 10, No. 1, 2010, pp. 61-83.

[2] F. M. Klaassen and W. Hes, "On the Temperature Coefficient of the MOSFET Threshold Voltages," Solid-State Electronics, Vol. 29, No. 8, 1986, pp. 787-789. doi:10.1016/0038-1101(86)90180-2

[3] [Online]. http://www.freedrinkingwater.com/water-education/qualit y-water-ph.htm.

[4] P. Bergveld, "Development of an Ion-Sensitive SolidState Device for Neurophysiologic Measurements," IEEE Transactions on Biomedical Engineering, Vol. 17, No. 1, 1970, pp. 70-71. doi:10.1109/TBME. 1970.4502688

[5] D. M. Wilson, S. Hoyt, J. Janata, K. Booksh and L. Obando, "Chemical Sensors for Portable, Handheld Field Instruments," IEEE Sensors Journal, Vol. 1, No. 4, 2001, pp. 256-274. doi:10.1109/7361.983465

[6] P. Bergveld, "Thirty Years of ISFETOLOGY What Happened in the Past 30 Years and What May Happen in the Next 30 Years," Sensors and Actuators B: Chemical, Vol. 88 , No. 1, 2000, pp. 1-20. doi:10.1016/S0925-4005(02)00301-5

[7] S. Jamasb, S. D. Collins and R. L. Smith, "A Physiccal Model for Threshold Voltage Insability in $\mathrm{SI}_{3} \mathrm{~N}_{4}$ Gate $\mathrm{H}^{+}$ Sensitive FETs," IEEE Tranactions on Electron Devices, Vol. 45 No. 6, 1998, pp. 1239-1245. doi:10.1109/16.678525

[8] L. J. Bousse, D. Hafeman and N. Tran, "Time Dependence of the Chemical Response of Silicon Nitride Surfaces," Sensors and Actuators B: Chemical, Vol. 1, No. 1-6, 1991, pp. 361-367.

[9] P. R. Barabash, R. S. C. Cobbold and W. B. Wlodarski, "Analysis of the Threshold Voltage and Its Temperature Dependence in Electrolyte-in Sulator-Semiconductor Field Effect Transistor," IEEE Transactions on Electronic Devices, Vol. 34, No. 6, 1987, pp. 1271-1282. doi:10.1109/T-ED.1987.23081

[10] N. S. Haron, M. K. B. Mahamad, I. A. Aziz and M. Mehat, "A System Architecture for Water Quality Monitoring System Using Wired Sensors," International Symposium on Information Technology, Kuala Lumpur, 26-28 August 2008, pp. 1-7. doi:10.1109/ITSIM.2008.4631927

[11] S. Martinoia and G. Massobrio, "A Behaviour MacroModel of the ISFET in SPICE," Sensors and Actuators B: Chemical, Vol. 62, No. 3, 2002, pp. 182-189. doi:10.1016/S0925-4005(99)00377-9

[12] B. Palan, F. V. Santos and J. M. Karam, "New ISFET Sensor Interface Circuit for Biomedical Applications," Sensors and Actuators B: Chemical, Vol. 57, No. 1-3, 1999, pp. 63-68. doi:10.1016/S0925-4005(99)00136-7

[13] C. H. Yang, W. Y. Chung, K. K. Lin, D. G. Pijanowska 
and W. Torbicz, "A Low-Power Telemetric System Design for ISFET-Based Sensor Array Applications," 16th European Conference on Circuit Theory and Design, Kraków, 1-4 September 2003, pp. 260-263.

[14] A. Morgenshtein, L. Sudakov-Boreysha and U. Dinnar, "CMOS Readout Circuitry for ISFET Micro-Systems," Sensors and Actuators B: Chemical, Vol. 97, No. 1, 2004, pp. 122-131. doi:10.1016/j.snb.2003.08.007

[15] R. E. G. Van Hal, J. C. T. Eijkel and P. Bergveld, “A Novel Description of ISFET Sensitivity with the Buffer Capacity and Double-Layer Capacitance as Key Parameters," Sensors and Actuators B: Chemical, Vols. 24-25, No. 1-3, 1995, pp. 201-205. doi:10.1016/0925-4005(95)85043-0

[16] Y.-H. Chang, Y.-S. Lu, Y.-L. Hong, S. Gwo and J. A. Yeh, "Highly Sensitive $\mathrm{pH}$ Sensing Using an Indium Nitride Ion-Sensitive Field-Effect Transistor," Vol. 11, No. 5, 2011, pp. 1157-1161.

[17] M. Lindquist and P. Wide, "New Sensor System for Drinking Water Quality," Proceedings the ISA/IEEE Sensors for Industry Conference, New Orleans, August 2004, pp. 30-34.

[18] R. M. Wen, Z. L. Zhu and S. L. Chen, "Preparation of high purity water with low concentration of dissolved oxygen (DO) and total organic carbon (TOC) for VLSI process," Proceeding of 6th International Conference

\section{Appendix}

Synthesis file

Release 10.1 - xst K.31 (nt)

Copyright (c) 1995-2008 Xilinx, Inc. All rights reserved.

--> Parameter TMPDIR set to C:/Users/Project Lab

Server/PASWAN/WEW/GH/xst/projnav.tmp

Total REAL time to Xst completion: 1.00 secs

Total CPU time to Xst completion: 0.13 secs

--> Parameter xsthdpdir set to $\mathrm{C}$ :/Users/Project Lab

Server/PASWAN/WEW/GH/xst

Total REAL time to Xst completion: 1.00 secs

Total CPU time to Xst completion: 0.13 secs

--> Reading design: Cell0.prj

*Synthesis Options Summary*

\begin{tabular}{|c|c|}
\hline ---- Source Parameters & \\
\hline $\begin{array}{l}\text { Input File Name } \\
\text { Input Format }\end{array}$ & $\begin{array}{l}\text { :"Cello.prj" } \\
\text { : mixed }\end{array}$ \\
\hline Ignore Synthesis Constraint File & $: \mathrm{NO}$ \\
\hline
\end{tabular}

---- Target Parameters
Solid-State and Integrated-Circuit Technology, Shanghai, 22-25 October 2001, p. 475-476.

[19] T.-Z. Qiao and L. Song, "The Design of Multi-Parameter Online Monitoring System of Water Quality Based on GPRS," 2010 International Conference on Multimedia Technology, Ningbo, 29-31 October 2010, pp. 1-3. doi:10.1109/ICMULT.2010.5631313

[20] B. R. Jean, "A Microwave Sensor for Steam Quality," IEEE Transactions on Instrumentation and Measurement, Vol. 57, No. 4, 2008, pp. 751-754. doi:10.1109/TIM.2007.913821

[21] J. C. Chou, H. M. Tsai, C. N. Shiao and J. S. Lin, "Study and Simulation of the Drift Behavior of Hydrogenated Amorphous Silicon Gate pH-ISFET," Sensors and Actuators B: Chemical, Vol. 62, No. 2, 2000, pp. 97-101. doi:10.1016/S0925-4005(99)00366-4

[22] S. Casans, A. E. Navarro, D. Ramirez, J. M. Espi, N. Abramova and A. Baldi, "Instrumentation System to Improve ISFET Behaviour," Proceeding of 19th IEEE Instrumentation and Measurement Technology Conference, Anchorage, 21-23 May 2002, pp. 1291-1294.

[23] S. Jamasb, "An Analytical Technique for Counteracting Drift in Ion-Selectivefield Effect Transistors (ISFETs)," IEEE Sensors Journal, Vol. 4, No. 6, 2004, pp. 795-801. doi:10.1109/JSEN.2004.833148

$\begin{array}{ll}\begin{array}{l}\text { Output File Name } \\ \text { Output Format }\end{array} & : \text { "Cell0” } \\ \text { Target Device } & : \text { AGC } \\ \text {--- Source Options } & : \text { Cell0 } \\ \text { Top Module Name } & : \text { YES } \\ \text { Automatic FSM Extraction } & : \text { Auto } \\ \text { FSM Encoding Algorithm } & : \text { YES } \\ \text { Mux Extraction } & : \text { YES } \\ \text { Resource Sharing } & \\ \text {--- Target Options } & : \text { YES } \\ \text { Add IO Buffers } & : \text { YES } \\ \text { MACRO Preserve } & : \text { YES } \\ \text { XOR Preserve } & : \text { YES } \\ \text { Equivalent register Removal } & \\ \text {--- General Options } & : \text { Speed } \\ \text { Optimization Goal } & : 1 \\ \text { Optimization Effort } & : \text { Cell0.lso } \\ \text { Library Search Order } & : \text { YES } \\ \text { Keep Hierarchy } & : \text { as_optimized } \\ \text { Netlist Hierarchy } & : \text { Yes } \\ \text { RTL Output } & : / \\ \text { Hierarchy Separator } & :<> \\ \text { Bus Delimiter } & \end{array}$




\begin{tabular}{|c|c|}
\hline Case Specifier & : maintain \\
\hline Verilog 2001 & : YES \\
\hline ---- Other Options & \\
\hline Clock Enable & : YES \\
\hline wysiwyg & : NO \\
\hline
\end{tabular}

* Final Report*

\begin{tabular}{|c|c|}
\hline \multicolumn{2}{|l|}{ Final Results } \\
\hline \multicolumn{2}{|c|}{ RTL Top Level Output File Name } \\
\hline \multicolumn{2}{|l|}{ Top Level Output File Name } \\
\hline \multicolumn{2}{|l|}{ Output Format } \\
\hline \multicolumn{2}{|l|}{ Optimization Goal } \\
\hline \multicolumn{2}{|l|}{ Keep Hierarchy } \\
\hline \multicolumn{2}{|r|}{ : Automotive 9500XL } \\
\hline \multicolumn{2}{|l|}{ Macro Preserve } \\
\hline XOR Preserve & $:_{\text {YES }}{ }^{: \text {YES }}$ \\
\hline Clock Enable & : YES \\
\hline wysiwyg & : NO \\
\hline \multicolumn{2}{|l|}{ Design Statistics } \\
\hline \# IOs & $: 4$ \\
\hline \multicolumn{2}{|l|}{ Cell Usage : } \\
\hline \# IO Buffers & $: 4$ \\
\hline \# IBUF & $: 1$ \\
\hline \# OBUF & $: 3$ \\
\hline \# Others & $: 16$ \\
\hline \# Capacitor & $: 1$ \\
\hline \# NMOS & $: 7$ \\
\hline \# PMOS & $: 4$ \\
\hline \# VoltageSource & $: 4$ \\
\hline
\end{tabular}

Total REAL time to Xst completion: 3.00 secs

Total CPU time to Xst completion: $2.50 \mathrm{secs}$

Total memory usage is 166132 kilobytes

Number of errors : $\quad 0$ (0 filtered)

Number of warnings : $\quad 0$ (0 filtered)

Number of infos : $\quad 0$ (0 filtered $)$
* T-Spice 13.02 Simulation Fri Oct 07 15:57:37 2011

C: $\backslash$ DOCUME $\sim 1 \backslash$ BPIT $\backslash$ LOCALS $\sim 1 \backslash$ Temp $\backslash$ Cell4.sp

* Command line: tspice $-\mathrm{O}$

$\mathrm{C}: \backslash \mathrm{DOCUME} \sim 1 \backslash \mathrm{BPIT} \backslash$ LOCALS $\sim 1 \backslash \mathrm{Temp} \backslash$ Cell4.out

C: $\backslash$ DOCUME $\sim 1 \backslash$ BPIT $\backslash$ LOCALS $\sim 1 \backslash$ Temp $\backslash$ Cell4.sp

* T-Spice Win32 13.02.20080516.01:34:09

TRANSIENT ANALYSIS

$\begin{array}{lcl}\text { Time }<\mathrm{s}> & \mathrm{v}(\text { in } 1)<\mathrm{V}> & \mathrm{v}(\text { out })<\mathrm{V}> \\ 0.000000 \mathrm{e}+000 & 0.0000 \mathrm{e}+000 & 0.0000 \mathrm{e}+000 \\ 1.250000 \mathrm{e}-010 & 1.2500 \mathrm{e}-001 & 9.5606 \mathrm{e}-002 \\ 6.786460 \mathrm{e}-010 & 6.7865 \mathrm{e}-001 & 5.4378 \mathrm{e}-001 \\ 9.554690 \mathrm{e}-010 & 9.5547 \mathrm{e}-001 & 7.8071 \mathrm{e}-001 \\ 1.093880 \mathrm{e}-009 & 1.0939 \mathrm{e}+000 & 8.9976 \mathrm{e}-001 \\ 1.316775 \mathrm{e}-009 & 1.3168 \mathrm{e}+000 & 1.0922 \mathrm{e}+000 \\ 1.600582 \mathrm{e}-009 & 1.6006 \mathrm{e}+000 & 1.3394 \mathrm{e}+000 \\ 1.922991 \mathrm{e}-009 & 1.9230 \mathrm{e}+000 & 1.6229 \mathrm{e}+000 \\ 2.303210 \mathrm{e}-009 & 2.3032 \mathrm{e}+000 & 1.9608 \mathrm{e}+000 \\ 2.758169 \mathrm{e}-009 & 2.7582 \mathrm{e}+000 & 2.3703 \mathrm{e}+000 \\ 3.296876 \mathrm{e}-009 & 3.2969 \mathrm{e}+000 & 2.8694 \mathrm{e}+000 \\ 3.931074 \mathrm{e}-009 & 3.9311 \mathrm{e}+000 & 3.4780 \mathrm{e}+000 \\ 4.678083 \mathrm{e}-009 & 4.6781 \mathrm{e}+000 & 4.2192 \mathrm{e}+000 \\ \text { 5.000000e-009 } & 5.0000 \mathrm{e}+000 & 4.5821 \mathrm{e}+000 \\ \text { * BEGIN NON-GRAPHICAL DATA } & \end{array}$

Power Results

vdd from time 0 to $5 \mathrm{e}-009$

Average power consumed -> 1.304338e-005 watts

Max power $1.635414 \mathrm{e}-005$ at time $5 \mathrm{e}-009$

Min power $0.000000 \mathrm{e}+000$ at time 0

* END NON-GRAPHICAL DATA*

* Parsing 0.00 seconds

* Setup 0.01 seconds

* DC operating point $\quad 0.00$ seconds

* Transient Analysis $\quad 0.01$ seconds

* Overhead 1.20 seconds

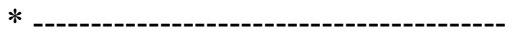

* Total 1.23 seconds

* Simulation completed with 1 Warning

* End of T-Spice output file 